\title{
Electrochemically active water repelling perfluorinated polyaniline films
}

\author{
Panagiotis Dallas ${ }^{* a b}$, Elena Tomšík ${ }^{c}$, Ruth Sang Jones ${ }^{\mathrm{a}}$, Albert Xiao ${ }^{\mathrm{a}}$, Eleanor Milnes-Smith ${ }^{\mathrm{d}}$, Nicole \\ Grobert $^{\mathrm{a}, \mathrm{e}}$ and Kyriakos Porfyrakis ${ }^{\mathrm{a}}$ \\ ${ }^{a}$ Institute of Nanoscience and Nanotechnology, NCSR Demokritos, Patriarhou Grigoriou \& \\ Neapoleos, 15310, Greece ${ }^{b}$ Department of Materials, University of Oxford, OX1 3PH, Oxford, United \\ Kingdom ${ }^{c}$ Institute of Macromolecular Chemistry AS CR, Heyrovsky Sq. 2, 16206 Prague 6, Czech \\ Republic ${ }^{d}$ Department of Chemistry, University of Oxford, United Kingdom ${ }^{e}$ Williams Advanced \\ Engineering, Grove, Oxfordshire, OX12 0DQ, UK \\ E-mail:p.dallas@inn.demokritos.gr; Tel: +2106503311
}

Keywords: Perfluorinated polyaniline, capacitance, hydrophobicity, microfibers

\begin{abstract}
Water repelling, perfluorinated, polyanilines and their composites with multi-wall carbon nanotubes are synthesized using interfacial polymerization in either flake-like or fibrillar shapes. This class of polyanilines exhibits electrochemical activity, capacitive behaviour, and a contact angle of 119$125^{\circ}$ with water. The addition of multi-wall carbon nanotubes facilitates the control of the polymer morphology and increases the specific capacitance of the material. We obtained microfibers or flakelike morphologies depending on the amount of multi-wall carbon nanotubes added in the organic phase and through cyclic voltammetry, impedance spectroscopy and galvanostatic charge-discharge, we evaluated the effect of the backbone geometry and the addition of nanotubes on the electrochemical properties of the composites and the pristine polymers. The capacitance of the linear 3-perfluoroctyl polyaniline is consistently better than the cross-linked 4-perfluoroctyl polyaniline, where the para position relative to the amine group is blocked by fluorocarbon chains.
\end{abstract}

\section{Introduction}

Hydrophobic conductive polymers are the optimum choice as solid contacts for ion selective electrodes. In this particular case, the hydrophobic surface is necessary in order to prevent the aqueous layer formation between the ion selective membrane and the solid contact. ${ }^{[1-2]}$ With respect to their water repelling properties, the perfluorinated materials comprise a unique class of compounds with extreme hydrophobicity ${ }^{[3]}$, unique solubility in supercritical carbon dioxide ${ }^{[4]}$, and increased fluorescence intensity and lower LUMO levels compared to their hydrocarbon counterparts. ${ }^{[5]}$ They have been applied as hydrophobic coatings in textile industry ${ }^{[6]}$, additives for enhanced oil recovery ${ }^{[7]}$ and molecular imaging and targeted therapeutics. ${ }^{[8]}$ In recent reports, the formation of fluorescent 
oligomeric species with tuneable fluorescence properties were reported for different monomers, specifically 3-perfluoroctyl polyaniline (denoted as 3PFA), and 4-perfluoroctyl polyaniline (denoted as 4PFA). ${ }^{[9-10]}$ The fluorescence of these oligomers can be tuned based on their protonation and oxidation states, and hence they were used as sensors for the colorimetric detection of organic acids and bases. ${ }^{[9]}$ Besides the soluble oligomers, during the polymerization at the interface of the two immiscible solvents, an insoluble polymer is formed that can easily be separated from the oligomers. Whilst the isolated oligomers present interesting, tuneable, red shifted photoluminescence properties with long Stokes shifts, the polymers isolated from the interface exhibit very weak fluorescence with extremely small Stokes shifts. ${ }^{[10]}$

Conventional polyaniline is a highly conductive polymer with remarkable magnetic and electronic transport behaviour that has been increasingly employed in applications such as supercapacitors [11], conductive coatings ${ }^{[12]}$, battery anodes, catalysts ${ }^{[13]}$, and as coatings for electromagnetic interference shielding. ${ }^{[14]}$ These properties are strongly dependent on the morphology of the material as also the assembly and packing of the polymer chains. Recently, the interaction of the polymer chains leading to the formation of nanofibers resulted in very high values of capacitance in polyaniline as measured from galvanostatic charge discharge measurements by Tomšík et al. [15]

In this report, we are expanding our previous work on the polymerization of 3-perfluoroctyl aniline (3PFA) and 4-perfluoroctyl aniline (4PFA) by focusing on the insoluble polymer films that form at the interface between the organic and the aqueous phase and their electrochemical properties. We synthesized four polymer films - 3PFA, 4PFA, and two 4PFA nanocomposites containing multi-wall carbon nanotubes (MWCNTs) synthesised using aerosol assisted chemical vapour deposition techniques. ${ }^{[16]}$ Different shapes are obtained through the interfacial polymerization, specifically fibers with a length of several microns and flake-like morphologies. The hydrophobic films exhibited a capacitive behaviour and the parameters characterising their electrical behaviour where obtained through Nyquist plots by applying different fitting models.

\section{Experimental Section}

2.1. Synthesis of carbon nanotubes. Multi-wall carbon nanotubes (MWCNTs) were synthesized using aerosol assisted chemical vapor deposition (AACVD) method modified from that reported by Meysami et al. [20] Briefly, a solution of $5 \mathrm{wt} . \%$ ferrocene (Aldrich 98\%) in toluene (Fluka, 99.7\%) was used in conjunction with an aerosol generator connected in series with a quartz tube located in a furnace. Argon (BOC 99.998\%) was passed through the system at $1000 \mathrm{sccm}$ as carrier gas. After an initial 15-minute Ar purging period at $800{ }^{\circ} \mathrm{C}$, the aerosol generator was turned on to produce an opaque and uniform aerosol, which was consequently drawn into the furnace by the carrier gas. The gas flow was maintained for 90 minutes, before the furnace cooled to room temperature. The multi-wall carbon nanotubes were collected by scraping the interior of the quartz tube. 
2.2. Synthesis of polymer composites. $1 \mathrm{mg}$ of multi-wall carbon nanotubes was dispersed in $10 \mathrm{ml}$ of chloroform after sonication to prepare the MWCNTs dispersion. The reaction vessel was a liquid scintillation vial (volume $20 \mathrm{~mL}$, vial size $28 \mathrm{~mm} \times 61 \mathrm{~mm}$, Sigma Aldrich) and no stirring took place during the reaction. Two different composites were synthesized with the following concentrations:

2.2.1. 4PFA-flakes: $300 \mathrm{mg}$ of monomer were added in $8 \mathrm{ml}$ of chloroform that contain $1 \mathrm{ml}$ of the MWCNTs dispersion. The aqueous phase consisted of $0.3 \mathrm{ml} \mathrm{HCl}$ and $720 \mathrm{mg}$ of $\mathrm{Na}_{2} \mathrm{~S}_{2} \mathrm{O}_{8}$ in $10 \mathrm{ml}$ of water.

2.2.2. 4PFA-fibers: $100 \mathrm{mg}$ of monomer in $8 \mathrm{ml}$ of MWCNTs chloroform dispersion and the aqueous phase contained $720 \mathrm{mg}$ of $\mathrm{Na}_{2} \mathrm{~S}_{2} \mathrm{O}_{8}$ and $0.3 \mathrm{ml} \mathrm{HCl}$ in $10 \mathrm{ml}$ of water. The polymerization proceeded for 2 days, the films were isolated with filtration and subsequently washed with water and chloroform. For the synthesis of pristine polymers, the concentration used for the synthesis of 4PFA-flakes was also applied for both the 3-perfluoroctyl aniline and the 4-perfluoroctyl aniline.

\section{Characterization techniques.}

3.1. Materials characterization. The JEOL $3000 \mathrm{~F}$ field emission transmission electron microscope (TEM), operating at $300 \mathrm{kV}$ acceleration voltage, was used for TEM imaging. Scanning electron microscope images were recorded on a JEOL 840F field emission scanning electron microscope, operating at $3 \mathrm{kV}$. Raman spectra were recorded on a LabRam Aramis Raman. To collect the FTIR spectra, we used a Varian Excalibur FTS 3500 spectrometer. The UV-Visible spectra were recorded in chloroform suspension on a Jasco spectrometer. The material was dispersed in toluene and the spectra were recorded in quartz cuvettes. Thermogravimetric analysis was conducted on the Perkin Elmer Pyris under air, with a heating rate of $10^{\circ} \mathrm{C} / \mathrm{min}$.

3.2. Electrochemical studies. All electrochemical measurements were performed in a three-electrode cell configuration. The polymer was deposited on carbon cloth (CC - Elat ${ }^{\circledR}$, Fuel Cells Etc. Co, USA, see Figure 4 for SEM images) by drop casting and the CC was used as a working electrode. A Pt sheet $\left(1.2 \mathrm{~cm}^{2}\right)$ was used as the counter electrode, and $\mathrm{Ag} / \mathrm{AgCl}(3 \mathrm{M} \mathrm{KCl})$ was used as the reference electrode. AUTOLAB PGSTAT302N potentiostat with FRA32M Module and Nova software 2.1 was used to record data. All electrochemical measurements were carried out at room temperature $\left(25^{\circ} \mathrm{C}\right)$. The electrolyte was a $6 \mathrm{M} \mathrm{H}_{3} \mathrm{PO}_{4}$ solution. The working electrode was immersed in the supporting electrolyte for few hours before the electrochemical studies.

3.3. Capacitance measurements. The galvanostatic charge/discharge measurements were performed in the potential window from $-200 \mathrm{mV}$ to $800 \mathrm{mV}$ vs $\mathrm{Ag} / \mathrm{AgCl}$ reference electrode with applied charge and discharge specific current from $0.06 \mathrm{~A} / \mathrm{g}$ to $0.5 \mathrm{~A} / \mathrm{g}$. The specific capacitance was calculated by the following equation (1):

$\mathrm{C}=(\mathrm{I} \times \mathrm{t}) /(\Delta \mathrm{V})$

where $\mathrm{I}$ is the applied discharge current $(\mathrm{A} / \mathrm{g}), \mathrm{t}$ is the discharge time $(\mathrm{s}), \Delta \mathrm{V}$ is the potential window without iR drop (V). 
3.4. Impedance spectroscopy. Electrochemical impedance spectroscopy (EIS) was performed in the frequency range from $10 \mathrm{kHz}$ to $0.1 \mathrm{~Hz}$ at constant potential $0.5 \mathrm{~V}$ with a $5 \mathrm{mV}$ amplitude. The KramersKronig test was applied to verify the obtained EIS data. The fitting was performed by using Nova software.

3.5. Cyclic voltammetry. Cyclic voltammetry was performed in a three-electrode cell configuration in the potential window from $-200 \mathrm{mV}$ to $800 \mathrm{mV}$ with scan rate $50 \mathrm{mV} / \mathrm{s}$.

\section{Results and discussion.}

\subsection{Formation of microfibers and nanoflakes.}

For the fabrication of the nanocomposites, the MWCNTs and the monomer were dispersed in the organic phase, in our case chloroform, while the oxidant and the hydrochloric acid were added in the aqueous phase. After 48 hours the reaction was complete and the insoluble polymer film that formed between the two solvents was isolated, washed with chloroform and air dried.

Fourier Transform Infrared (FTIR) spectra were employed for the identification of the oxidation state and structure of the materials. This is rather crucial for this class of materials, since their oxidation and protonation state and their backbone geometry dictate their capacitive and electronic transport behaviour. The spectra for both the 3PFA and the 4PFA are shown in Figure 1. The two polymers are growing in two different configurations, depending on the position of the perfluorinated groups as can be seen in Figure $1 \mathrm{~b}$. This is further evidenced by the different splitting of the $-\mathrm{C}=\mathrm{C}$ - vibrational bands in the 1400-1600 $\mathrm{cm}^{-1}$ FTIR region (Figure 1a-II). The 3PFA exhibits a linear backbone, while the blocked para position in the case of 4PFA, as shown in Figure 1b, leads to an extensive cross-linking. According to previous works on linear polyaniline the absorption bands in the region $3400-2800 \mathrm{~cm}^{-1}$ relates to nitrogen-containing groups. A broad absorption band at frequencies higher than $2000 \mathrm{~cm}^{-1}$ is considered a characteristic of conductive forms of polyaniline specifically in the region of 3200-2600 $\mathrm{cm}^{-1}$. ${ }^{[16]}$ These broad bands originate from secondary amine $-\mathrm{NH}-$ and protonated imine $-\mathrm{NH}^{+}=$, and it is an overtone of hydrogen bonding chains. ${ }^{[17-18]}$ The FTIR bands at 1540 and $1497 \mathrm{~cm}^{-1}$, are assigned to quinoid and benzenoid ring-stretching of the polymer backbone in the linear 3PFA, in a similar manner to the conventional polyaniline, while for the cross-linked 4PFA are appearing at 1542 and 1520 $\mathrm{cm}^{-1}$. The band at $1620 \mathrm{~cm}^{-1}$ is due to a Raman-active $-\mathrm{C}=\mathrm{C}-$ ring-stretching vibration. ${ }^{[19]}$ The FTIR of the pristine and the composite polymer of the 4-perfluoroctyl polyaniline are almost identical, signaling that the addition of nanotubes is not affecting the structure of the polymer. 


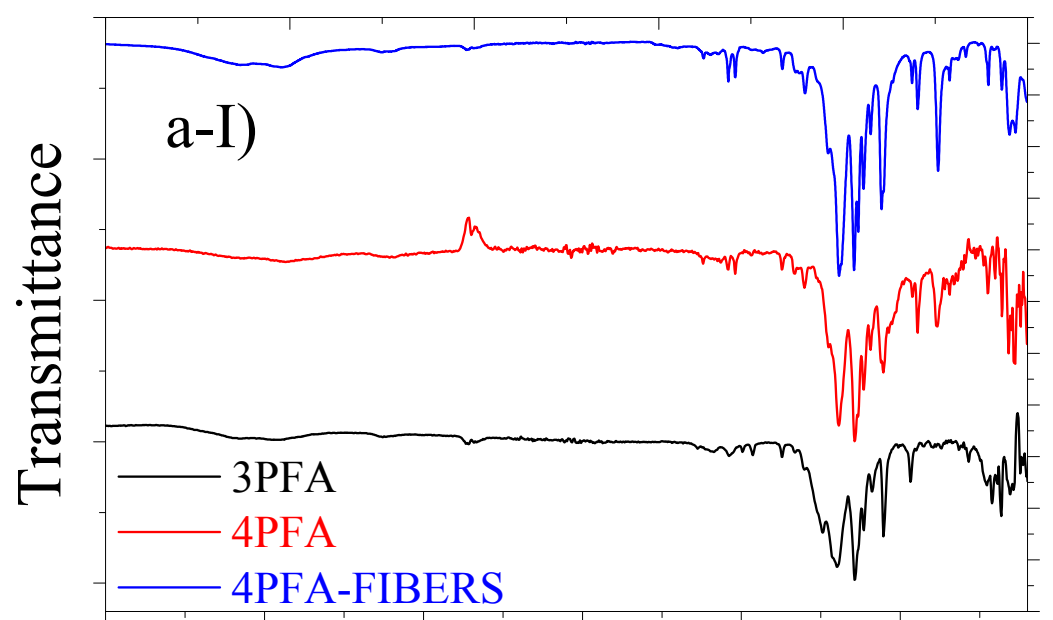

$350030002500200015001000-1$

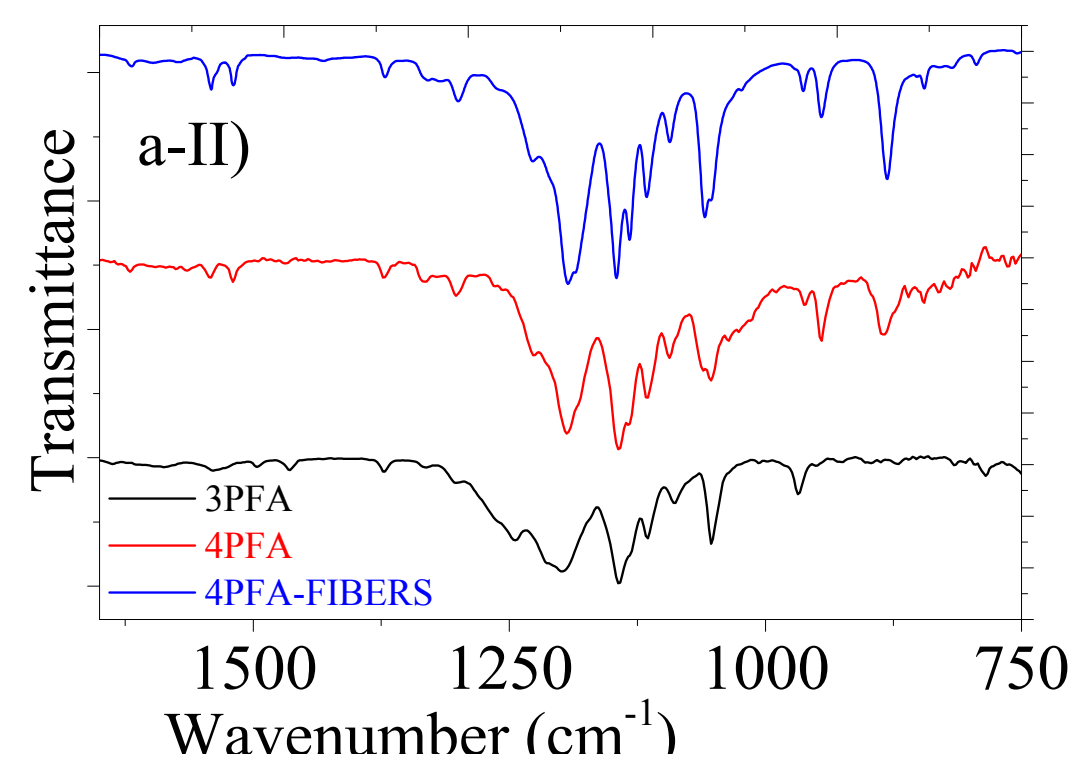

3-perfluoroctyl polyaniline<smiles>Cc1ccc(N=C2C=CC(=Nc3ccc([NH+]=C4C=CC(=N)C=C4C(F)(F)F)cc3C(F)(F)F)C=C2C(F)(F)F)cc1C(F)(F)F</smiles>

4-perfluoroctyl polyaniline

4-perfluoroctyl polyaniline
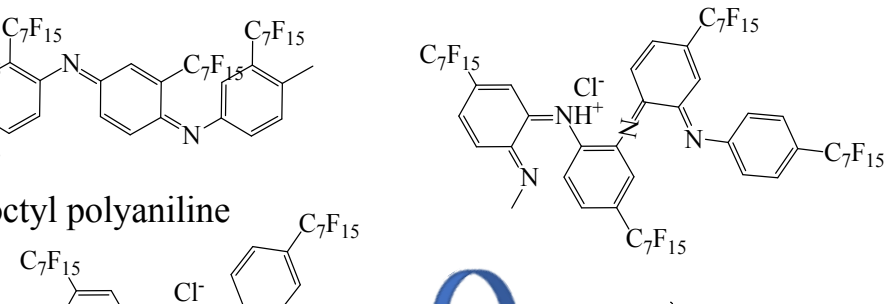

b)
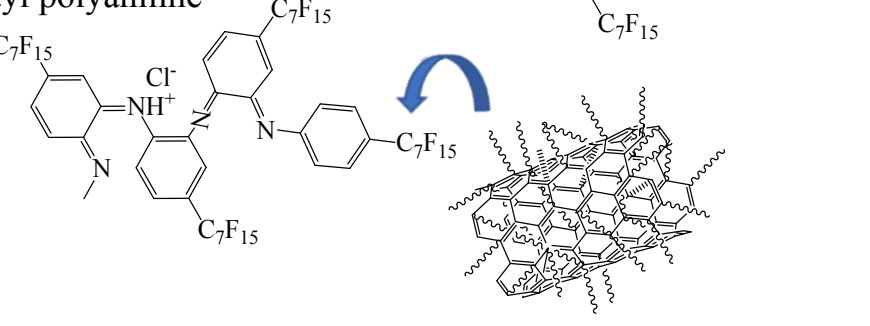
Figure 1. a-I) FTIR spectra of 3PFA (black), 4PFA (red) and 4PFA-fibers (blue). Magnification at the $\mathrm{N}-\mathrm{H}$ and C-N region. a-II) Magnification at the region where the $-\mathrm{N}-\mathrm{H}, \mathrm{C}-\mathrm{N}$ and C-F vibrational modes appear. b) The proposed structure of the 3PFA and 4PFA.

Since perfluorinated materials have unique and unusual solubility properties ${ }^{[20-21]}$ and demonstrate increased hydrophobicity, we measured their contact angles with water on 4PFA films as derived from the interface. These static contact angle measurements are shown in Figure 2 and consistent values of $119-125^{\circ}$ were observed for three different films, proving the strong repellent properties of the films towards water bearing in mind the common concept of hydrophobicity. A material can be defined as superhydrophobic when the contact angle is $>140^{\circ}$, hence the films reported here still possess a minor affinity towards water. ${ }^{[22]}$ Here, we have to emphasize that this value is significantly higher compared to other conductive polymers that have been used as hydrophobic solid contacts, such as perfluorooctanesulfonate doped polypyrrole that showed a contact angle of $97^{\circ} .{ }^{[1-2]}$ In that case the hydrophobic fluorocarbon was acting as a counterion and was not covalently bonded on the polymer backbone as in the perfluorinated polyanilines. The materials appear to be rather brittle, hence we did not attempt to record their mechanical properties.

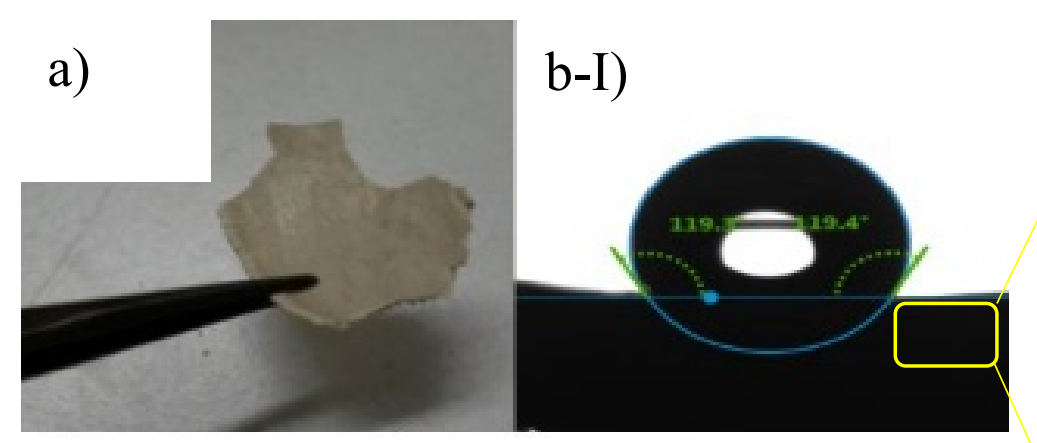<smiles>FC(F)C(F)C(F)CC(F)C(F)(F)F</smiles><smiles>FC(F)C(F)CC(F)C(F)C(F)F</smiles>

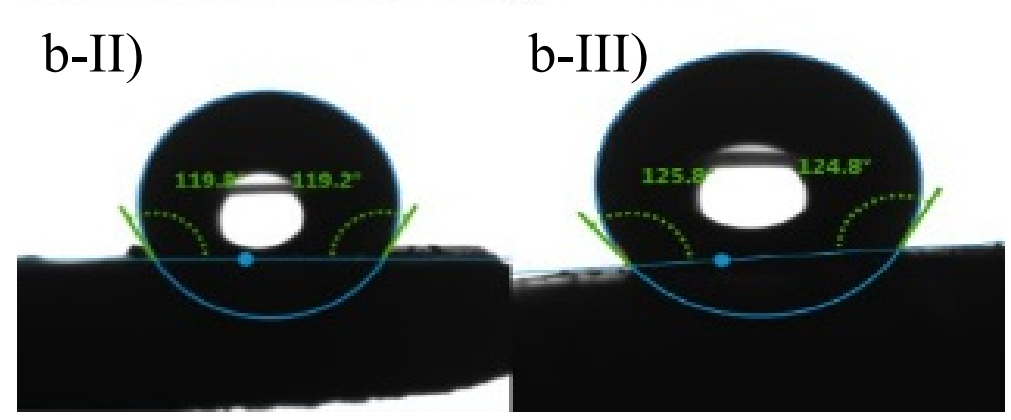<smiles>FC(F)C(F)CC(F)C(F)C(F)F</smiles>

Figure 2. a) The film can be easily isolated from the interface. bI-III) Water contact angle measurements in three different spots using the pendant drop method. The 4PFA films demonstrate a hydrophobic behaviour with contact angle against water at $119-125^{\circ} \mathrm{C}$.

According to optical spectroscopy studies, the polymers appear to have a small band gap and are subject to solvatochromic effects as evidenced by UV-visible spectroscopy studies. In Figure 3a, 
we present the UV-visible spectra of the pristine 3PFA and 4PFA in acetone, tetrahydrofuran (THF) and N,N' dimethylformamide (DMF) solutions. The spectra do not resemble the conventional polyaniline due to the presence of electron withdrawing C-F groups, and most likely to a lower molecular weight so they mostly resemble oligomeric structures. ${ }^{[9]}$ One interesting effect that we need to mention for clarity is the colour change of the polymers upon slow drying and removal of the chloroform immediately after the completion of the polymerization. The powders shift from a pale pink to white once the solvent is completely removed, see the images in Figure 3b. The reason for this colour change is still unclear, but we speculate it originates from variable intrachain interactions.

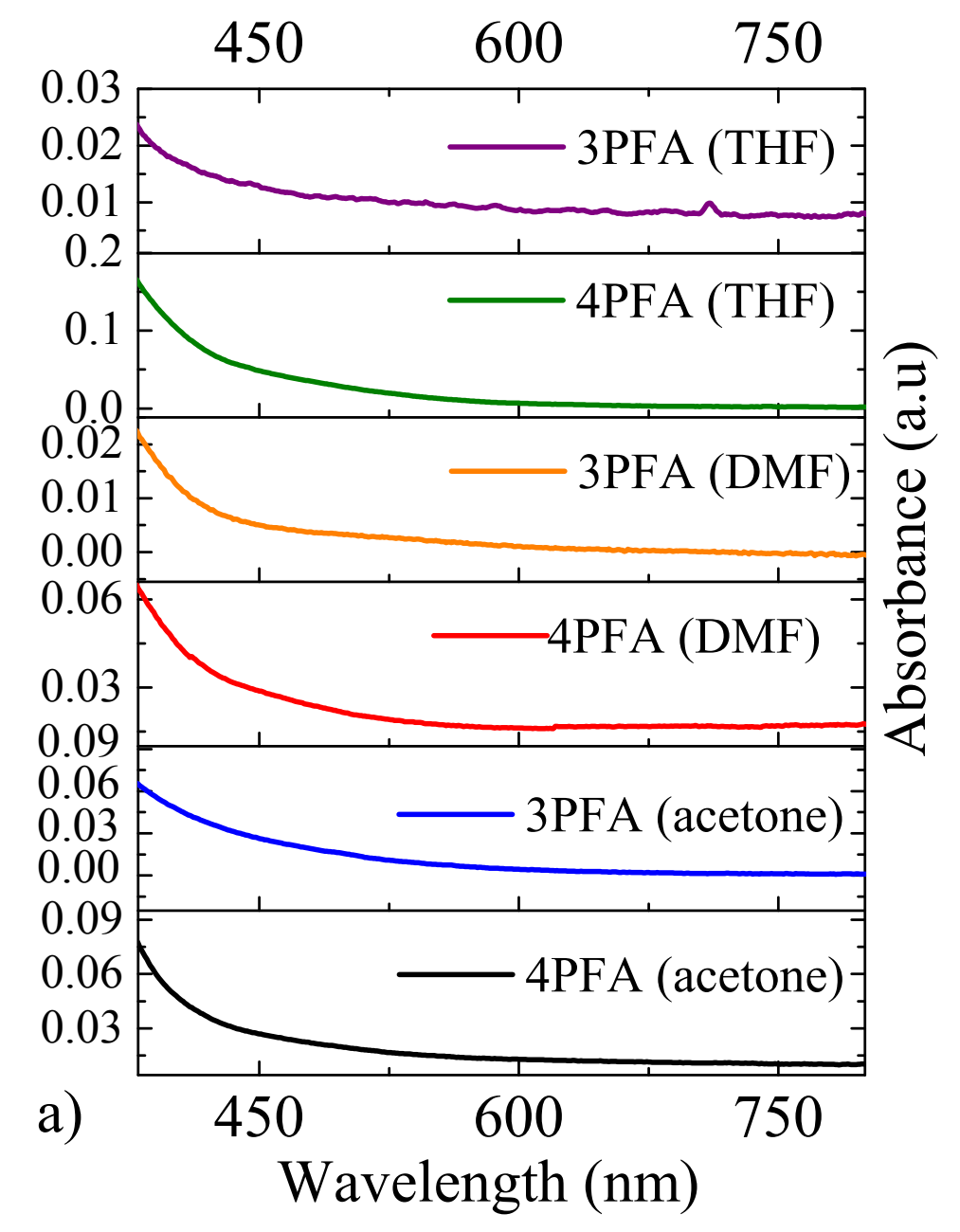



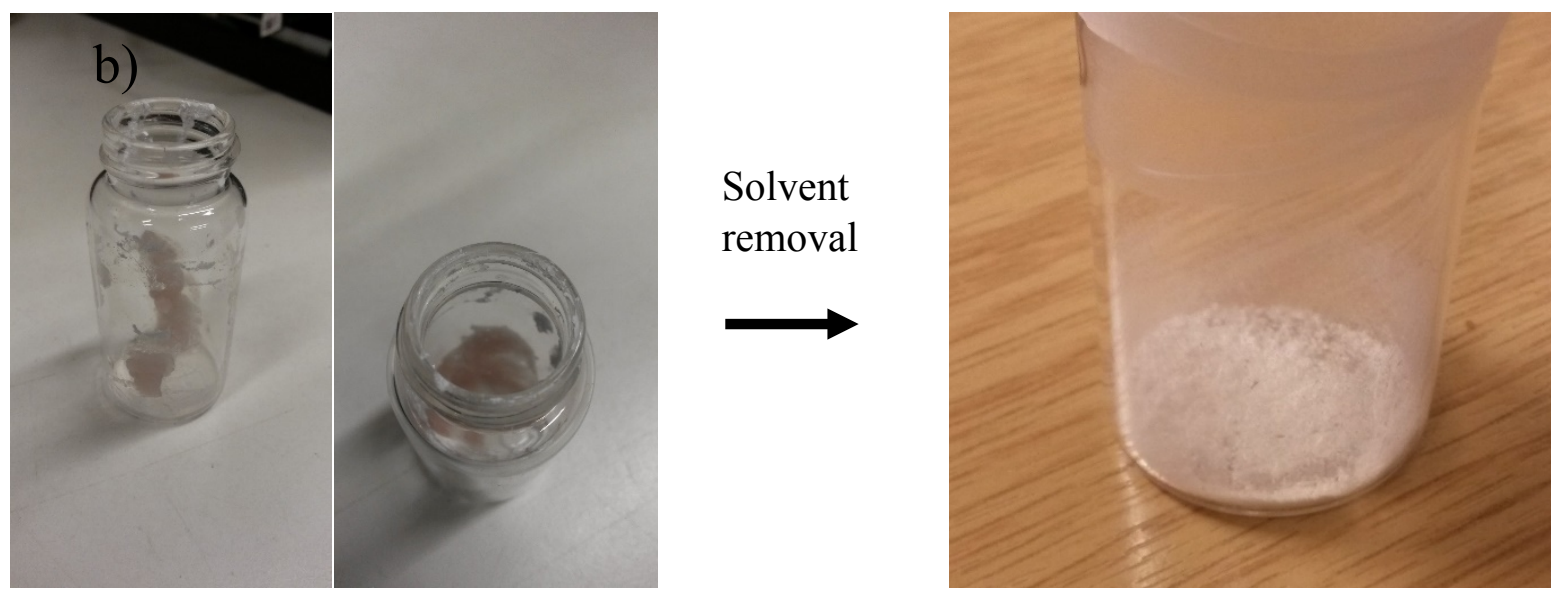

Figure 3. a) UV-Visible spectra of 3PFA and 4PFA samples recorded in acetone, N,N' dimethylformamide and tetrahydrofuran. b) The polymer powder changes from a pale pink to white colour as the chloroform evaporates.

Scanning and transmission electron micrographs (SEM and TEM) of the two composites are shown in Figures 4a-c and S4. If the polymerization process is performed using relatively high monomer concentrations (as in the case of 4PFA-flakes), the material grows in flake-like shapes similar to the pristine 3PFA polymer previously reported by Dallas et al. ${ }^{[9]}$ Such film formation is an uncommon feature for conductive polymers that are synthesized through interfacial polymerization. Conventional polyaniline tends to form fibrillar morphologies with diameters of a few tenths of $\mathrm{nm}{ }^{[23]}$, while depending on the reaction conditions other morphologies may be obtained as well [24]. In contrast, the unique solubility parameters of the perfluorinated monomers and the growth in a restricted space, e.g. a two-dimensional interface, force the polymer to assemble in these flat film-like morphologies (4PFAflakes). However, if the polymerization is performed with a high MWCNT:monomer ratio (comp.B), the carbon nanotubes act as templates and force the polymers to self-assemble into highly uniform fibres (4PFA-fibers) (Figure 4b). Depending on the amount of carbon nanotubes added to the 4PFA interfacial polymerization reaction, different morphologies can be obtained. The evolution of polyaniline nanostructures is rather complex even in the case of conventional, linear, polyaniline. ${ }^{[25]}$ A detailed analysis of the formation mechanism of the nanofibers would require a completely new manuscript that would focus on observation of oligomers and polymers during different reaction times. Through thermogravimetric analysis which presents two clear decomposition steps the first assigned to the polymer and later at higher temperatures to carbon nanotubes, we calculated a MWCNT loading of 20.6 wt $\%$, with the 4PFA-fibers exhibiting a higher thermal stability compared to the 4PFA-flakes with the decomposition initiated at higher temperatures. The graphs are presented in Figure 4e. 

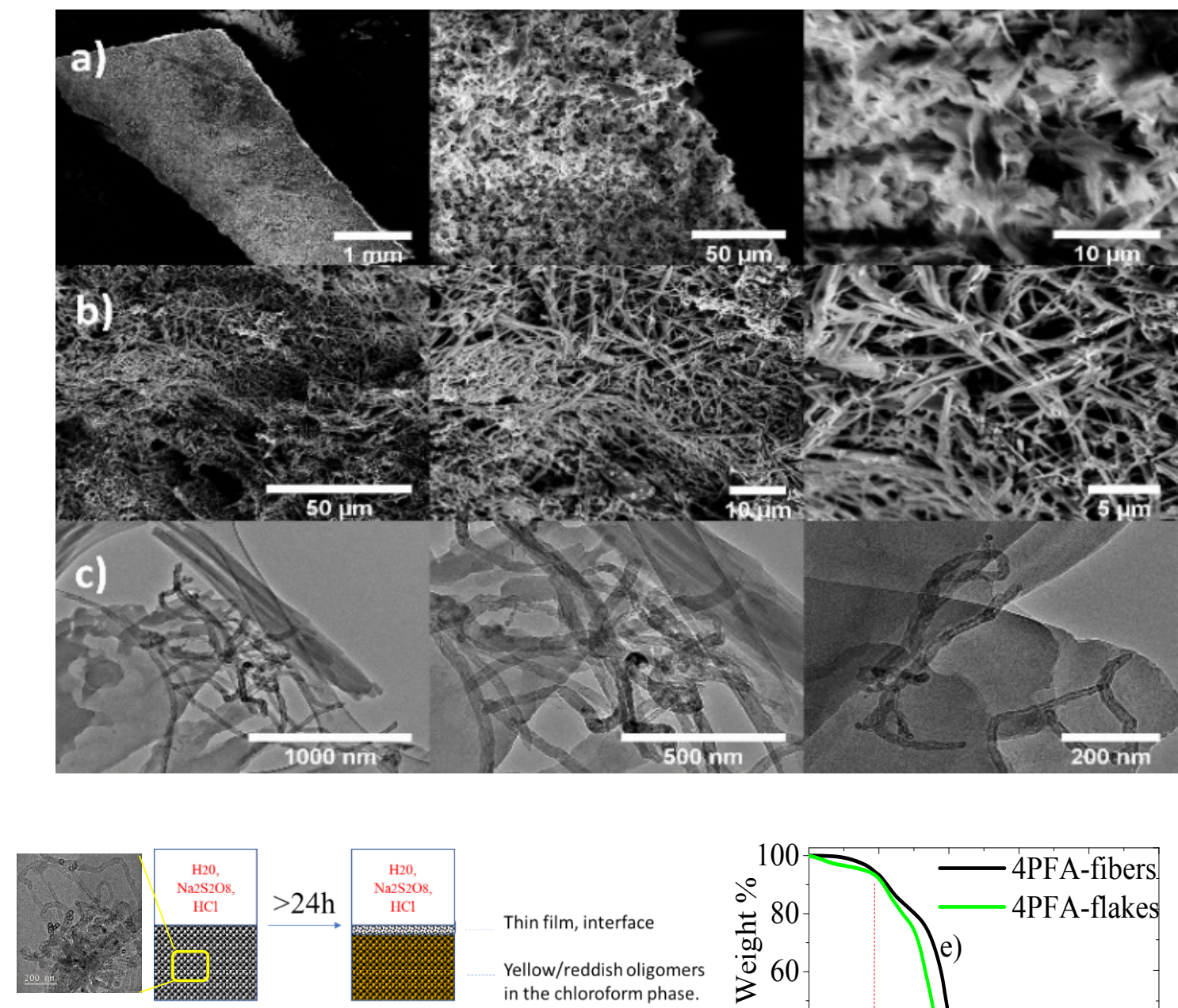

Thin film, interface

Yellow/reddish oligomer in the chloroform phase.

d)
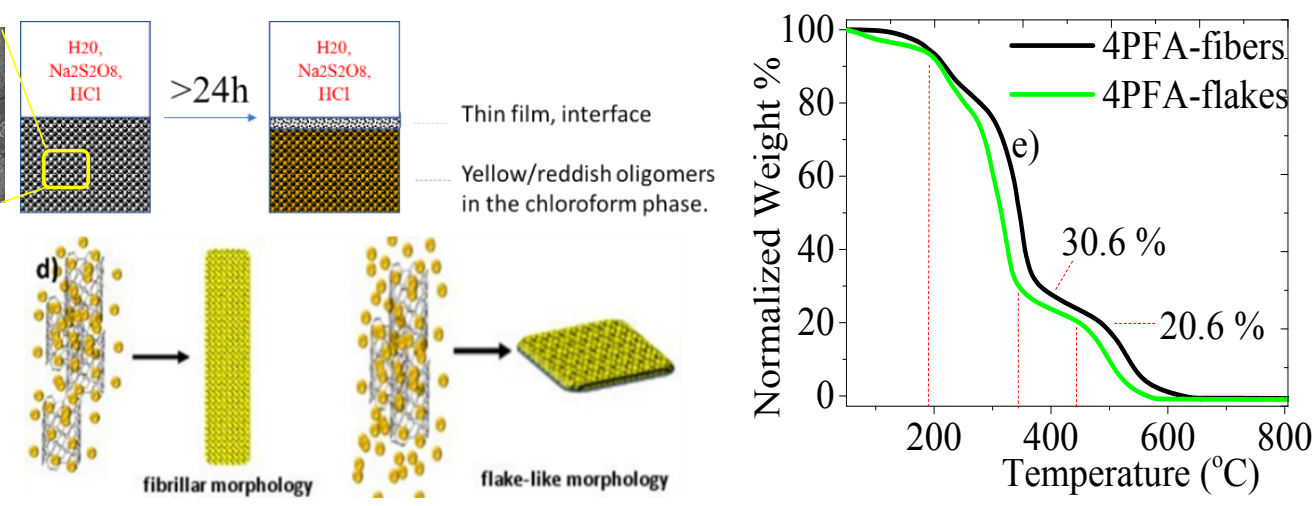

Figure 4. SEM images of the a) MWCNT composite films with the nanotubes embedded in flake-like 4PFA, and b) fibrillar 4PFA-MWCNT composite. c) TEM images of 4PFA-fibers nanocomposite drop casted from toluene suspensions. d) Schematic representation of the assembly of carbon nanotubes inside microfibers (4PFA-fibers) or flake-like polymer (4PFA-flakes). The yellow spheres represent the monomer. e) Thermogravimetric analysis of 4PFA-MWCNT-fibers (black) and 4PFA-MWCNT-flakes (green).

\subsection{Capacitive properties and electrochemical behavior.}



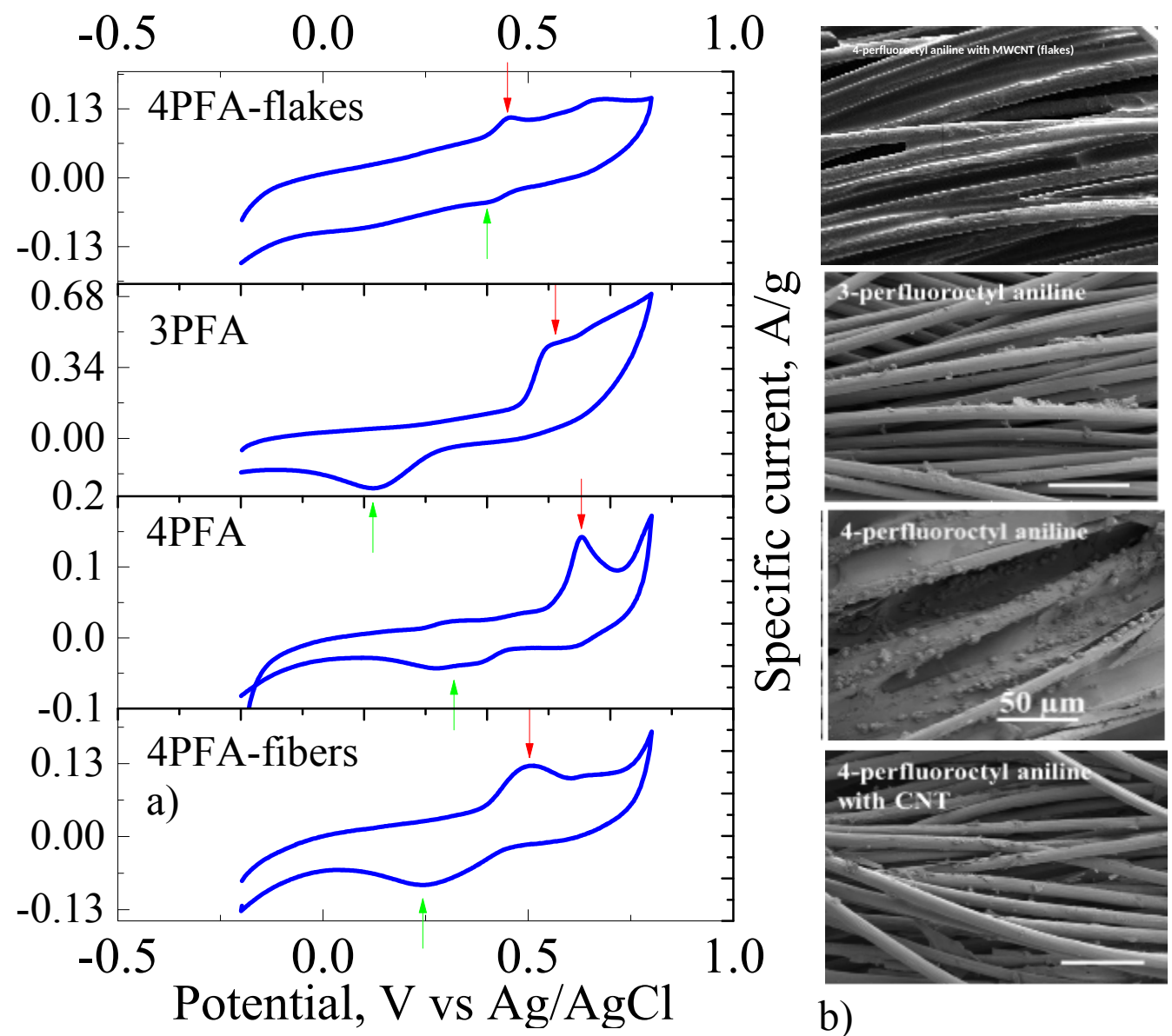

b)

Figure 5. a) Cyclic voltammetry graphs for 3-perfluoroctyl polyaniline, 4-perfluoroctyl aniline and the fibrillar and flake-like composites of 4-perfluoroctyl polyaniline with carbon nanotubes and the corresponding SEM images of the polymers deposited on carbon cloth. (b) All scale bars are $50 \mu \mathrm{m}$, and the images were recorded under the same magnification and resolution.

We subsequently proceeded with the evaluation of the electrochemical properties of the 3PFA, 4PFA, and 4PFA-MWCNT (both flake-like and fibrillary morphologies) samples initially through cyclic voltammetry studies. The materials were deposited on carbon cloth and cyclic voltammetry measurements were performed in acidic aqueous solutions. SEM images of the samples on carbon cloth are shown in Figure 5. From the voltammographs shown in Figure 5, the 3PFA appears to have lower oxidation potential $(0.54 \mathrm{~V}$ vs $\mathrm{Ag} / \mathrm{AgCl})$ compared to sample $4 \mathrm{PFA}(0.63 \mathrm{~V} \mathrm{vs} \mathrm{Ag} / \mathrm{AgCl})$. However, when carbon nanotubes were added during the polymerization process, the oxidation potential shifted downward $(0.45 \mathrm{~V}$ for flakes and $0.51 \mathrm{~V}$ for fibers $v \mathrm{Ag} / \mathrm{AgCl})$, which proves that the carbon nanotubes incorporated into the polymer facilitate charge transfer as was also confirmed by Electrochemical impedance spectroscopy (EIS). The reduction potential is the lowest for 3PFA (0.11 V vs $\mathrm{Ag} / \mathrm{AgCl})$ and the highest for $4 \mathrm{PFA}(0.3 \mathrm{~V}$ vs $\mathrm{Ag} / \mathrm{AgCl})$. It must be mentioned that the smallest separation between oxidation and reduction potentials was observed for 4PFA with carbon nanotubes. This observation can be explained by the fact that the carbon nanotubes facilitate the charge propagation 
and charge transfer during the electrochemical measurements. On the other hand, 3PFA showed the highest specific current, which is attributable to the linear backbone of the polymer. Galvanostatic charge-discharge studies were performed with the films deposited on carbon cloth. The graphs and the parameters obtained can be seen in Figure 6 and Table 1 respectively. The specific capacitance for 4PFA is lower compared to 3PFA, likely due to extensive cross link chains on the former while the latter appears to be linear. Sun et al studied the capacitance behaviour of linear and branched polyaniline. [26] Similarly to our finding, the linear polyaniline has higher capacitance compared to the branched polyaniline. They also observed a massive increase of the capacitance of the branched polymer after subsequent cycles, which was attributed to the rupture of the branched phenazine-like units to linear fragments.

\begin{tabular}{|c|c|c|c|c|}
\hline \multicolumn{5}{|c|}{$\underline{\text { 3PFA }}$} \\
\hline I, specific current $A / g$ & iR drop, $\mathbf{V}$ & $\tau_{\text {disch }}, \mathbf{s}$ & $\begin{array}{c}\text { Specific } \\
\text { capacitance, } \\
\text { F/g }\end{array}$ & $\begin{array}{c}\text { Coulombic efficiency, } \\
\tau_{\text {disch }} \tau_{\text {char }}(100 \%)\end{array}$ \\
\hline 0.13 & 0.0045 & 9.24 & 1.2 & 64 \\
\hline 0.22 & 0.0085 & 4.72 & 1.04 & 55 \\
\hline 0.4 & 0.013 & 2.14 & 0.9 & 66 \\
\hline 0.44 & 0.015 & 1.9 & 0.85 & 64 \\
\hline \multicolumn{5}{|c|}{ 4PFA-MWCNT-fibers } \\
\hline 0.16 & 0.0149 & 14.03 & 2.3 & 45 \\
\hline 0.33 & 0.028 & 4.8 & 1.6 & 56 \\
\hline 0.5 & 0.043 & 2.51 & 1.3 & 60 \\
\hline 0.65 & 0.054 & 1.54 & 1.06 & 57 \\
\hline \multicolumn{5}{|c|}{$\underline{\text { 4PFA }}$} \\
\hline 0.06 & 0.005 & 11.29 & 0.68 & 62 \\
\hline 0.13 & 0.012 & 3.66 & 0.48 & 67 \\
\hline 0.19 & 0.015 & 2.48 & 0.48 & 71 \\
\hline 0.38 & 0.029 & 0.4 & 0.4 & 64 \\
\hline 0.5 & 0.036 & 0.38 & 0.38 & 61 \\
\hline \multicolumn{5}{|c|}{ 4PFA-MWCNT-flakes } \\
\hline 0.12 & 0.0051 & 38.03 & 4.6 & 89 \\
\hline 0.2 & 0.008 & 7.45 & 1.5 & 72 \\
\hline 0.33 & 0.013 & 4.45 & 1.5 & 63 \\
\hline
\end{tabular}


Table 1. Specific capacitance, specific current, iR drop and coulombic efficiency values.

As seen from Figure 6b and Table 1, 3PFA has a higher specific capacitance compared to 4PFA. The introduction of MWCNT inside the 4PFA chains, leads to a nearly three and six times increase of the specific capacitance, at the same specific current charge and discharge for the fibers and flakes 4FPA-CNT composites respectively. It must be emphasized that even if the perfluorinated polyanilines are hydrophobic polymers, all of them possess a very small iR drop (Table 1), which is most probably connected with the morphology of the deposited film. Another notable feature is that the Coulombic efficiency is higher for 4PFA compared to 3PFA, the reason for which is to be further investigated, with the highest value to be observed for the 4PFA-CNT-flakes composite.
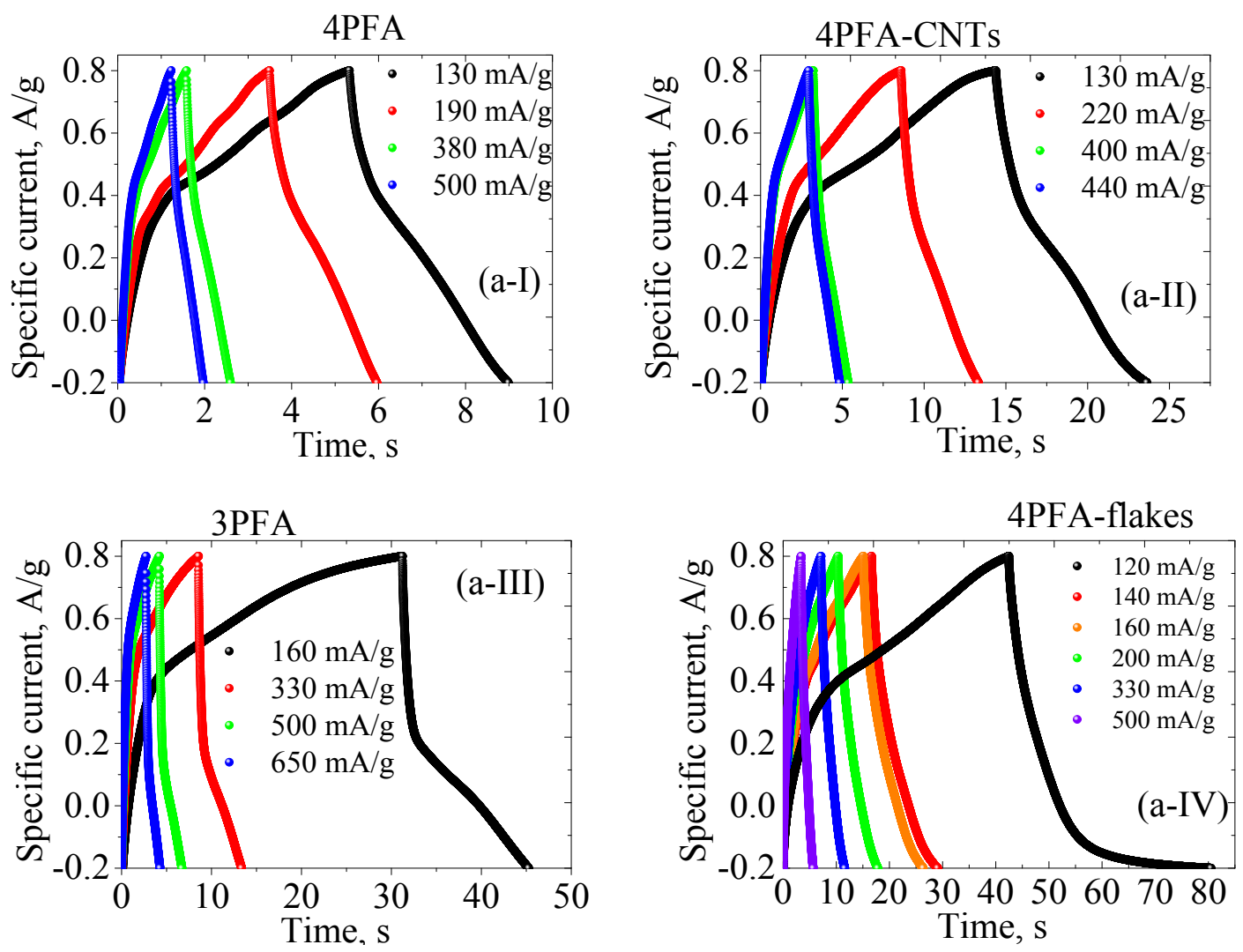


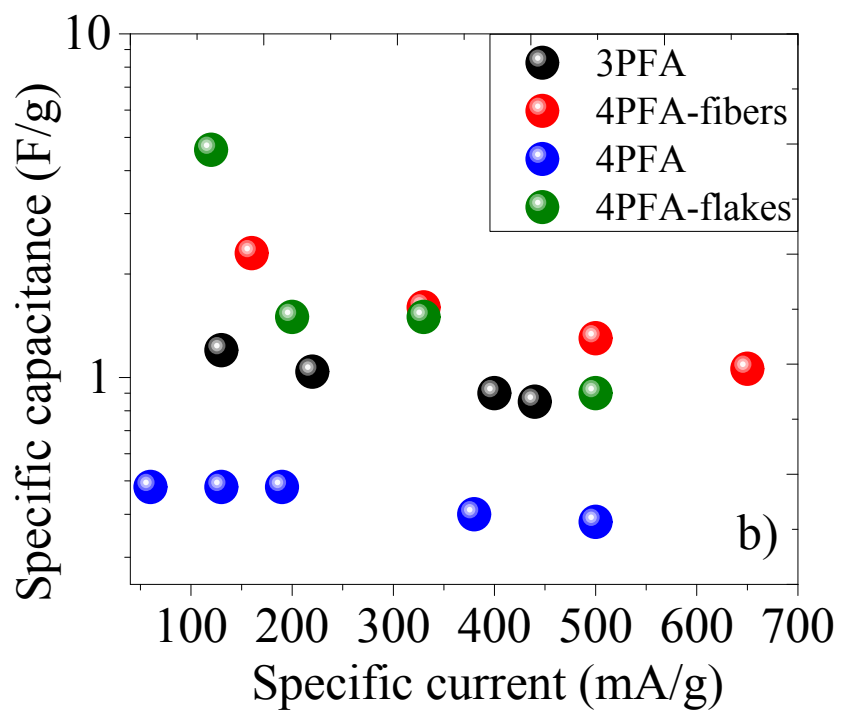

Figure 6. a) Galvanostatic charge -discharge measurements for 4PFA (i), 4PFA-CNT fibers (II), 3PFA (III) and 4PFA-CNT flakes (IV). b) The graph summarizes the specific capacitance and specific current values for all samples.

\subsection{Electrical response: impedance spectroscopy and fitting models.}

Electrochemical impedance spectroscopy is the method of choice for characterizing the electrical behaviour of systems in which the overall system behaviour is determined by strongly coupled processes, each proceeding at a different rate. EIS data are commonly analysed by fitting to an equivalent electrical circuit model, the two models used by us are shown in Figure 8. ${ }^{[27]}$ The fitting parameters are summarized in Table 2. In our polymeric systems we also observed a double layer capacitance, a capacitive behaviour that exists at the interface between an electrode and its surrounding electrolyte (denoted as $\mathrm{C}_{\mathrm{dl}}$ ). Furthermore, ion diffusion can also create an additional impedance, which is known as the Warburg impedance. EIS studies were performed for the polymers and composite samples at a constant potential $0.5 \mathrm{~V}$ vs $\mathrm{Ag} / \mathrm{AgCl}$.

The solution resistance is similar in all measurements since the same electrolyte was used (Figure 7). A Nyquist plot approaching an ideal capacitor ( $c a .90^{\circ}$ phase shift at low frequencies), represented by an almost straight line at low frequency is observed for the 4PFA-fibers sample. As the measurements demonstrated the composite samples (4PFA-fibers and 4PFA-flakes) and liner 3PFA are following a linear fitting, opposing to the 4PFA that shows clear tendency to form a semicircle. Moreover, for the 4PFA and 3PFA, a slight dependence of impedance on the higher frequencies was observed, which could be explained by the presence of charge transfer resistance (Figure 7a) ${ }^{[26]}$. On the other hand, the impedance of the 3PFA, 4PFA-fibers and 4PFA-flakes did not possess such dependency (Figure 7b). The absence of the charge transfer resistance could be connected to the molecular structure of the 3PFA sample, and the presence of MWCNTs in the 4PFA sample. 

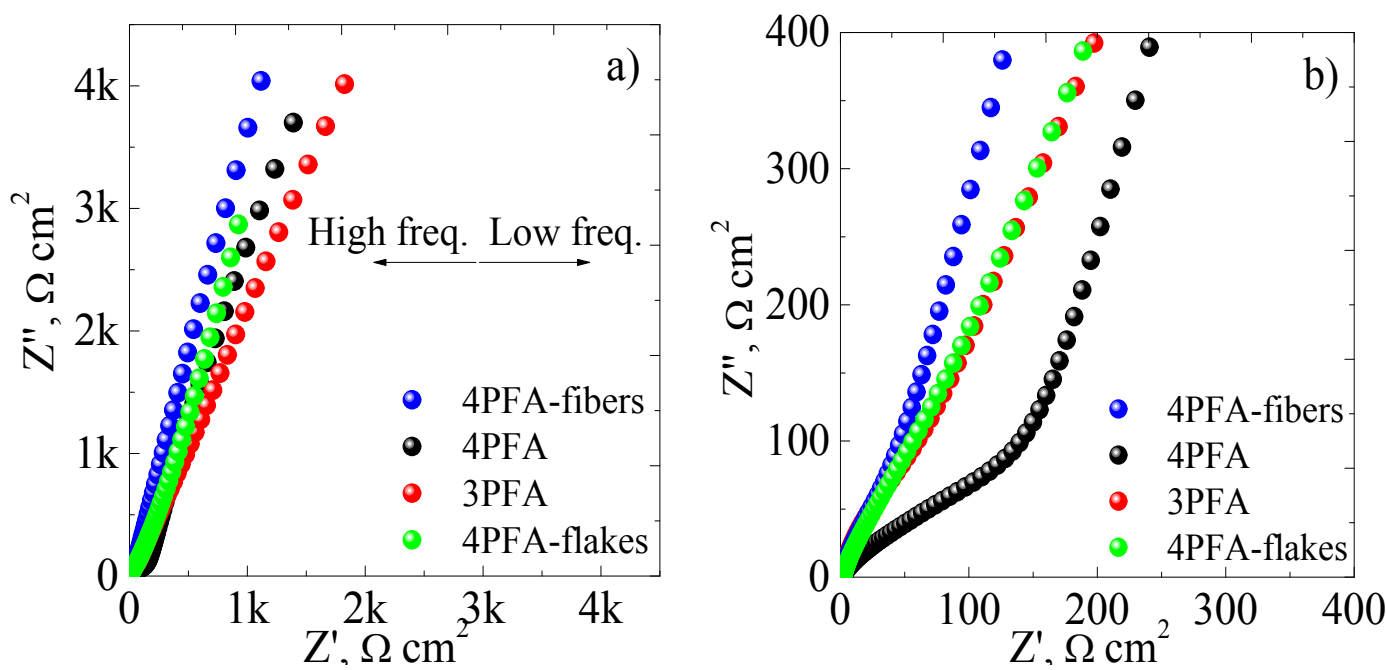

Figure 7. a) Nyquist plots of the two pristine perfluorinated polyanilines and the fibrillar and flake-like composites deposited on carbon cloth, measured in aqueous solution in three-electrode cell configuration. b) Close-up of (a).

We applied two different fitting models to identify the separate processes that take place during EIS measurements. (Figure 8). The estimated errors for the fitting parameters can be seen in Table 3. The first model was applied to 4PFA and the second to 4PFA-fibers, 4PFA-flakes and 3PFA. The 4PFA has a faradaic capacitance $(502 \mathrm{mF} / \mathrm{g})$, with a value similar to the one obtained from the galvanostatic charge discharge measurements. The samples 3PFA, 4PFA-fibers and flakes exhibit imperfect capacitance in the form of constant phase element, suggesting that the effective capacitance and 'real' resistance are increasing as the frequency decreases. In electrochemical systems with rough surfaces or a distribution of reaction rates for the electrochemical processes in the substrate mass appears the constant phase element (CPE). ${ }^{[28]} \mathrm{CPE}$ is an imperfect capacitance meaning that the capacitance does not become constant at low frequency - the effective capacitance and 'real' resistance are increasing as the frequency decreases. ${ }^{[29]}$
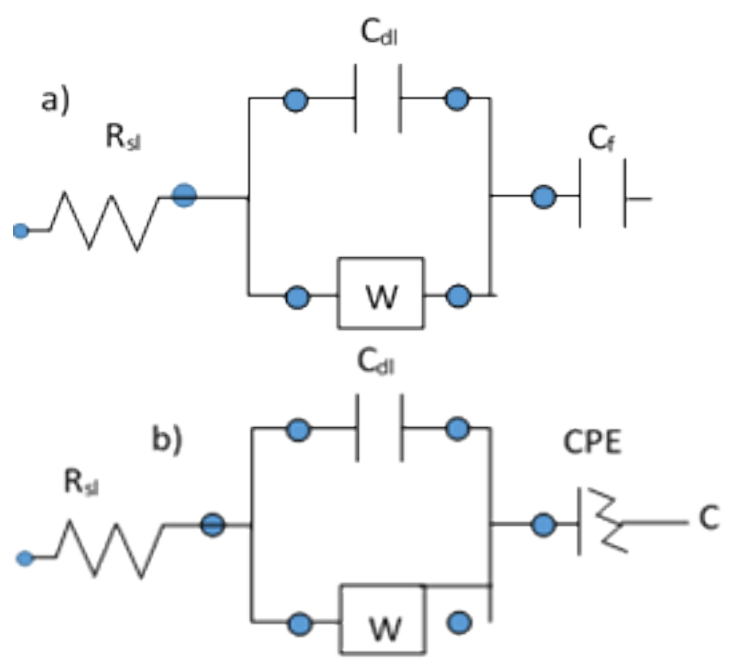
Table 2.

\begin{tabular}{|c|c|c|c|c|c|c|}
\hline Sample & $\begin{array}{c}\mathbf{R}_{\mathrm{sl}}, \\
\mathbf{\Omega} \times \mathbf{c} \\
\mathbf{m}^{\mathbf{2}}\end{array}$ & $\underset{\mu \mathrm{F} / \mathbf{c m}^{2}}{\mathrm{C}_{\mathrm{dl}}}$ & $\begin{array}{c}\mathbf{W}, \\
\mu M_{h o} \times \mathbf{s}^{0.5}\end{array}$ & 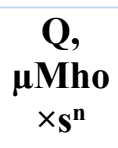 & n & $\chi^{2}$ \\
\hline $\begin{array}{l}\text { 4PFA- } \\
\text { fibers }\end{array}$ & 1.36 & 61 & 582 & 154 & 0.94 & 0.14167 \\
\hline 4PFA & 1.31 & 8.4 & 502 & - & - & 0.12077 \\
\hline 3PFA & 1.27 & 30 & 286 & 172 & 0.89 & 0.14784 \\
\hline $\begin{array}{l}\text { 4PFA- } \\
\text { flakes }\end{array}$ & 1.8 & 43 & 595 & 246 & 0.87 & 0.11016 \\
\hline
\end{tabular}

Table 3.

\begin{tabular}{|c|c|c|}
\hline \multicolumn{3}{|c|}{ 4PFA } \\
\hline Element & Estimated error (\%) & $\mathbf{x}^{2}$ \\
\hline $\mathrm{R}_{\mathrm{sl}}$ & 1.146 & \multirow[t]{4}{*}{0.12077} \\
\hline $\mathrm{C}_{\mathrm{dl}}$ & 2.231 & \\
\hline $\mathrm{W}$ & 3.845 & \\
\hline $\mathrm{C}_{\mathrm{f}}$ & 1.201 & \\
\hline \multicolumn{3}{|c|}{ 4PFA-fibers } \\
\hline Element & Estimated error (\%) & $\mathbf{x}^{2}$ \\
\hline $\mathrm{R}_{\mathrm{sl}}$ & 1.123 & \multirow[t]{4}{*}{0.14167} \\
\hline $\mathrm{C}_{\mathrm{dl}}$ & 2.421 & \\
\hline $\mathrm{W}$ & 3.741 & \\
\hline $\mathrm{CPE}$ & 1.298 & \\
\hline \multicolumn{3}{|c|}{ 4PFA-flakes } \\
\hline Element & Estimated error (\%) & $\mathbf{x}^{2}$ \\
\hline $\mathrm{R}_{\mathrm{sl}}$ & 1.125 & \multirow[t]{4}{*}{0.11} \\
\hline $\mathrm{C}_{\mathrm{dl}}$ & 3.918 & \\
\hline $\mathrm{W}$ & 2.630 & \\
\hline $\mathrm{CPE}$ & 1.224 & \\
\hline \multicolumn{3}{|c|}{ 3PFA } \\
\hline Element & Estimated error $(\%)$ & $\mathbf{x}^{2}$ \\
\hline $\mathrm{R}_{\mathrm{sl}}$ & 1.135 & \multirow[t]{3}{*}{0.14784} \\
\hline $\mathrm{C}_{\mathrm{dl}}$ & 2.894 & \\
\hline $\mathrm{W}$ & 3.415 & \\
\hline
\end{tabular}


Figure 8. Fitting models for a) 4-PFA $R_{s l}\left(C_{d l} W\right) C_{f}$ and b) for the other samples $R_{s l}\left(C_{d l} W\right) C_{f .}$. Table 2. The parameters obtained from the fitting are summarized in Table 1. Where: $\mathrm{R}$ - ideal resistance; $\mathrm{C}$ - ideal (double layer) capacitance; W - Warburg impedance; and Q - constant phase element (CPE).

Table 3. Fitting parameters with estimated errors (\%) for the EIS measurements.

The double layer capacitance is the highest for the 4PFA-MWCNT-fibers. This behaviour should be expected, since the MWCNTs will contribute to a surface increase for the composite material. The proposed models in these systems are imperfect, since the sum of squares of the relative residuals ( $\chi 2$ value) is relatively high. This observation is attributed to an imperfect fitting at low frequencies compared to the fitting at higher frequencies. Based on this observation, we conclude that more complex processes are taking place at low frequencies. When comparing the fitted EIS data for Warburg impedance, 3PFA shows the lowest value, which means that the diffusion of ions is holding a minor role in the electrochemical process compared to the 4PFA and 4PFA-MWCNT samples. This observation could be related to the morphology of the deposited films and more importantly with the structure of the polymer. Based on the results of galvanostatic charge-discharge and EIS we could conclude that 3PFA is suitable polymer as a solid contact for ion selective electrodes. Detailed investigation of the EIS of the polymers at different applied constant potentials, various film thickness and electrolytes is in progress and will be reported elsewhere.

\section{Conclusions}

In summary, we presented the electrochemical properties of hydrophobic fibers or flakes consisting of perfluorinated polyanilines with or without the addition of multi-wall carbon nanotubes. The materials may be used in niche applications were the combination of electrochemical activity, conductivity and hydrophobicity is necessary, for example as solid contacts for ion selective electrodes. The capacitance of the linear polymer, 3PFA, is consistently better compared to that of the branched, cross-linked, 4PFA. We assign this difference to be emergent from the inherent structures of the two polyaniline monomers, whereby 3PFA has a linear backbone that is more facilitating to charge transfer. While, in general, polyaniline is a polymer dispersible in water, the anchoring perfluorinated chains are rendering the polymer hydrophobic hence it can be used in sensor and solid contacts applications, where a resistance to humidity is desirable. ${ }^{[30]}$ Overall, these electrochemically active materials present numerous advantages, including the straightforward preparation of films, the low cost of the procedure, the high yield and ease of purification.

\section{Acknowledgements}


The authors would like to acknowledge Prof. Susan Perkin for providing access to the contact angle measurements apparatus. We are grateful to the Hellenic Foundation for Research and Innovation (1468), EPSRC (EP/K030108/1, the Pathways to Impact grants, and Impact Acceleration Accounts), the Royal Society, and the European Research Council (ERC-2016-PoC-754748 OxfordNano; ERC-2015PoC-680559 CONDUCT; ERC-2012-PoC-309786 DEVICE; ERC-2009-StG-240500 DEDIGROWTH) for their financial support

\section{References}

[1] N. He, S. Papp, T. Lindfors, L. Hofler, R.-M. Latonen, R. E. Gyurcsanyi, Anal.Chem. 89 (2017) 2598-2605

[2] M. Guzinski, J.M. Jarvis, P. D’Orazio, A. Izadyar, B. D. Pendley, E. Lindner. Anal. Chem. 89 (2017) 8468

[3] J.C.Biffinger, H.W.Kim, S.G.DiMagno. ChemBioChem. 5 (2004) 622-627

[4] J.Eastoe, S. Gold, D.C. Steytler. Langmuir 22 (2006) 9832

[5] U. Giovanella, C. Botta, F. Galeotti, B. Vercelli, S. Battiato, M. Pasini. J. Mater. Chem. C. 1 (2013) 5322-5329

[6] G.Lv, L.Wang, S.Liu, S.Li. Anal.Sci. 25 (2009) 425

[7] A. O.Al-Amodi, U.A. Al-Mubaiyedh, A.S.Sultan, M.S.Kamal, I.A. Hussein. Can.J.Chem.Eng. 94 (2016) 454

[8] T.D.Trand. et al. Int.J.Nanomedicine 2 (2007) 515

[9] P.Dallas, I.Rašović, K.Porfyrakis. J.Phys.Chem.B. 120 (2016) 3441

[10] P.Dallas, I.Rasovic, T.Puchtler, R.A. Taylor, K. Porfyrakis. Chem.Comm. 53 (2017) 2602

[11] D.Jin, Z.Qin, Y.Shen, T.Li, L.Ding, Y.Chen, Y.Zhang. J.Sol.State Electr. 22 (2018) 1227

[12] X.M.Li, D.Reinhoudt, M.Crego-Calama. Chem.Soc.Rev. 36 (2007) 1350-1368

[13] H.Yang, T.Song, L.Liu, A.Devadoss, F.Xia, H.Han, H.Park, W.Sigmund, K.Kwon, U.Paik. J.Phys.Chem.C. 117 (2013) 17376

[14] H.R. Tantawy, D.E. Aston, J.R. Smith, J.L. Young. ACS Appl. Mater. Interfaces 5 (2013) 4648-4658

[15] E.N. Tomšík, O. Kohut, I. Ivanko, M. Pekárek, I. Bieloshapka, P. Dallas. J.Phys.Chem.C. 122 (2018) 822-8030

[16] S.S. Meysami, F. Dillon, A.A. Koos, Z. Aslam, N. Grobert. Carbon 8 (2013) 2-9

[17] Z. Rozlívková, M. Trchová, I. Šeděnková, M. Špírková, J. Stejskal. Thin Solid Films 519 (2011) 5933-5941

[18] S. Wang, A.A. Rogachev, M.A. Yarmolenko, A.V. Rogachev, J. Xiaohong, M.S. Gaur, P.A. Luchnikov, O.V. Galtseva, S.A. Chizhik. Appl.Surf.Sci. 428 (2018) 1070-1078

[19] J. Mihály, S. Sterkel, H.M. Ortner, L. Kocsis, L. Hajba, É. Furdyga, J. Mink. Croatica Chimica Acta. 79 (2006) 497-501 
[20] J.M.DeSimone, E.E.Maury, Y.Z.Menceloglu, J.B.McClain, T.J.Romack, J.R.Combers.

Science 265 (1994) 356-359

[21] V.H.Dalvi, P.J.Rossky. Proc.Nat.Acad.Sci.USA. 107 (2010) 10603-10607

[22] K-Y.Lew. J.Phys.Chem.Lett. 5 (2014) 686-688

[23] J. Huang, S. Virji, B.H. Weiller, R.B. Kaner. J.Am.Chem.Soc. 125 (2003) 314

[24] C. Oueiny, S. Berlioz and F. X. Perrin. Phys.Chem.Chem.Phys. 18 (2016) 3504

[25] M. Trchová, I. Šeděnková, E.N. Konyushenko, J. Stejskal, P. Holler, G. Ćirić-Marjanović. J. Phys. Chem. B 110 (2006) 199461

[26] J.Sun, L.Chen. React.Funct.Pol. 138 (2019) 55

[27] B.E. Conway, Electrochemical Supercapacitors: Scientific Fundamentals and Technological Applications. New York, 1999.

[28] G. Lang, G. Inzelt. Electrochimica Acta. 36 (1991) 847-854

[29] M.C.E. Bandeira, R. Holze. Microchim Acta. 156 (2007) 125-131

[30] M.Guzinski, J.M.Jarvis, P.D’Orazio, A.Izadyar, B.D. Pendley, E.Lindner. Anal.Chem. 89 (2017) 8468-8475 